\title{
Catalase activity of abzymes in blood of patients with schizophrenia
}

\author{
Evgeny Ermakov ${ }^{1,2,3}$, Smirnova L.P. ${ }^{3}$, Buneva V.N. ${ }^{1,2}$, Ivanova S.A. ${ }^{3}$ \\ 1. Novosibirsk State University, Novosibirsk, Russia \\ 2. Institute of Chemical Biology and Fundamental Medicine, Novosibirsk, Russia \\ 3. Mental Health Research Institute of the Tomsk National Research Medical Center, Tomsk, Russia \\ Author for correspondence: evgeny_ermakov@majl.ru \\ WCP19-1644
}

\begin{abstract}
Abzymes are immunoglobulins that have catalytic functions, including redox-active ones. Abzymes with antioxidants activities have been detected in the serum of healthy donors [Tolmacheva et al., $J M R$ 2015]. It is possible, that abzymes can also be generated in patients with schizophrenia.

In this work we showed that abzymes of schizophrenia patients have antioxidant, i.e. catalase activity, that this activity belongs directly to antibodies and that this activity depends on the presence of metal ions with variable valency.
\end{abstract}

\section{Evidence that catalase activity belongs to abzymes:}

1. The absence of impurities of other proteins in the preparations of the analyzed $\operatorname{lgG}$ according to the results of SDS-PAGE and MALDI-MS (data not shown).

2. Detection of catalase activity after gel filtration under acidic conditions and after SDS-PAGE in fractions corresponding to $\lg G$ (Pic. 1 A-F).

Oxidative stress is known to be actively involved in the pathogenesis of schizophrenia. We assume that redox-active abzymes can compensate for redox imbalance in schizophrenia. However, the contribution of abzymes to the work of the antioxidant defense system remains to be seen.

\section{Proof that that the directly abzymes of patients with} schizophrenia possesses catalase activitv
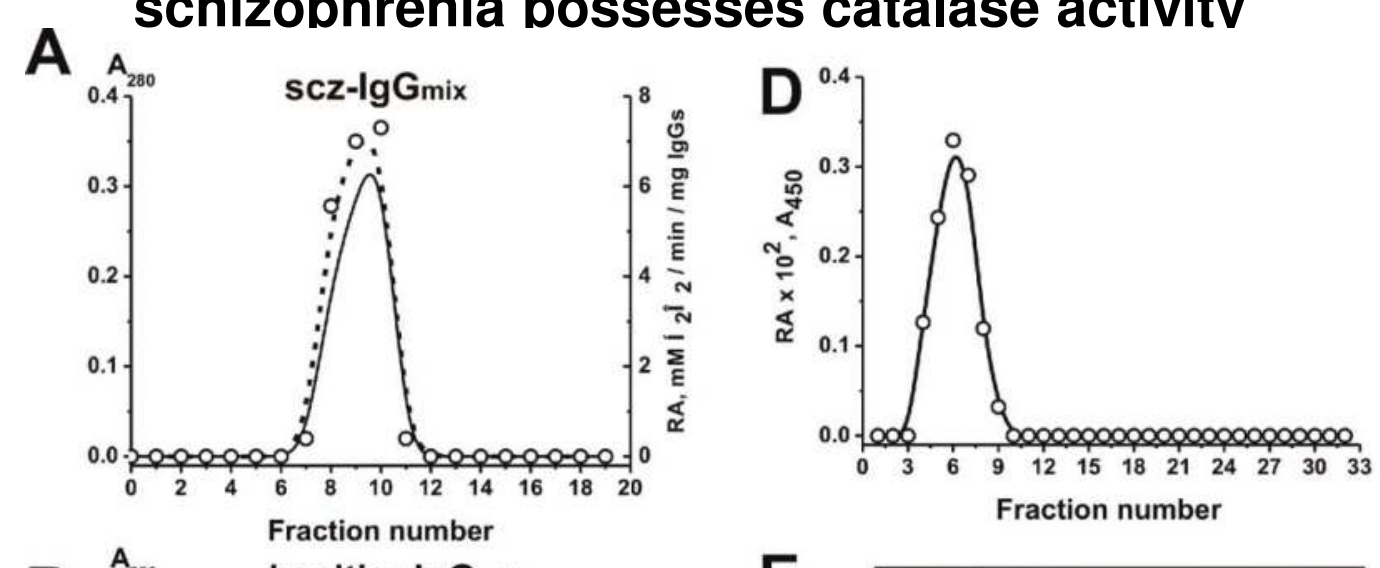

B
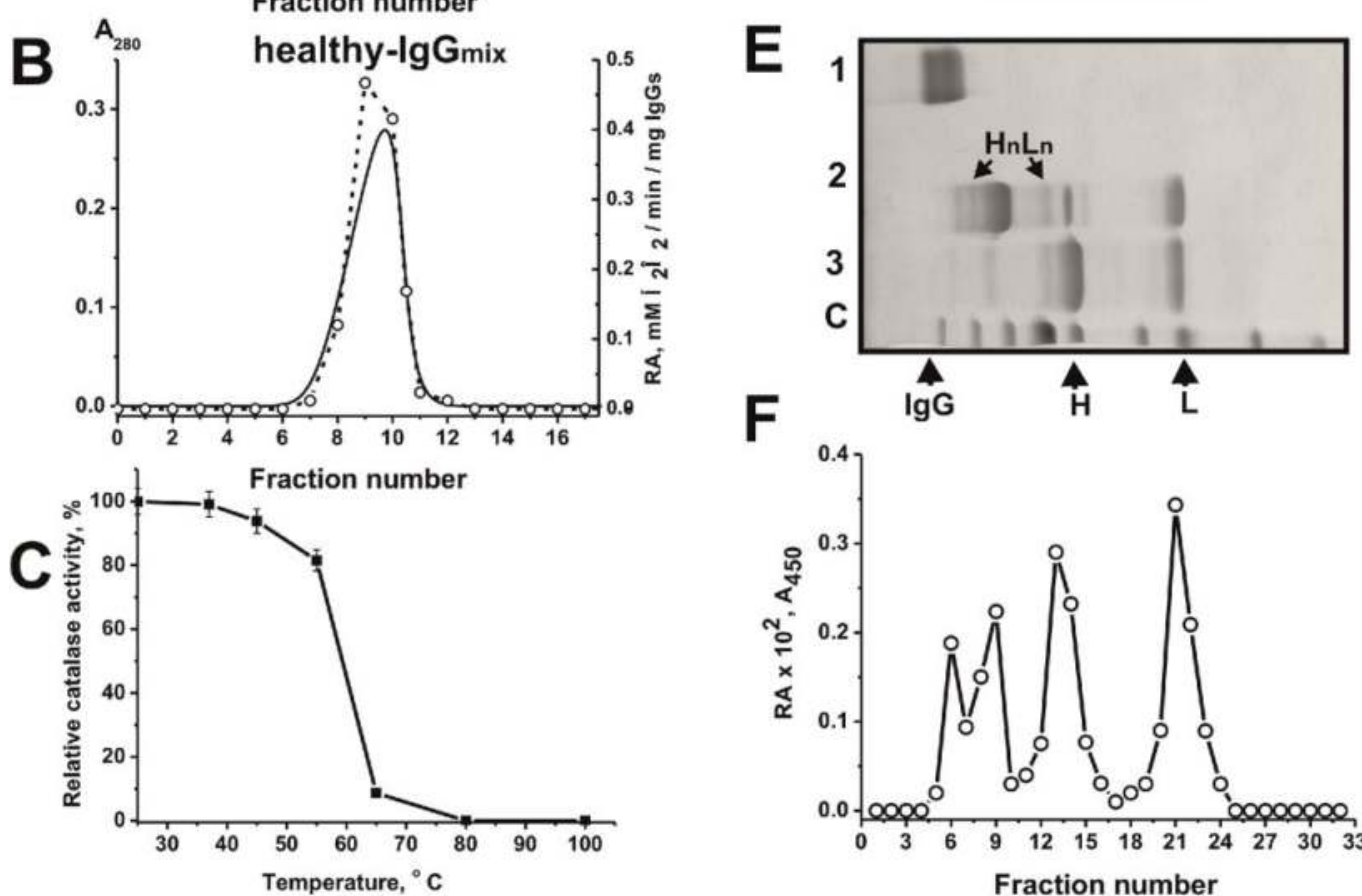

A, B - The gel-filtration of $\lg G$ under acidic conditions $(\mathrm{pH} 2,6)$ and analysis of catalase activity in obtained fractions.

$C$ - Analysis of the thermal stability of IgG preparation.

D, E, F - SDS-PAGE analysis of catalase activity of $\mathrm{lgG}$.

\section{Main Results:}

1. The IgG abzymes in serum of patients with schizophrenia possess higher catalase activity as compared of healthy donors.

2. Catalase activity belongs to abzymes

3. Catalase activity depends on the presence of metal ions.

4. The kinetic parameters of abzymes was significantly lower than that of classical catalase enzymes.

Abzymes with catalase activity can perform an antioxidant function and protect the body from oxidative stress.

\section{Abzymes of patients with schizophrenia have higher catalase} activity than abzymes of healthy donors

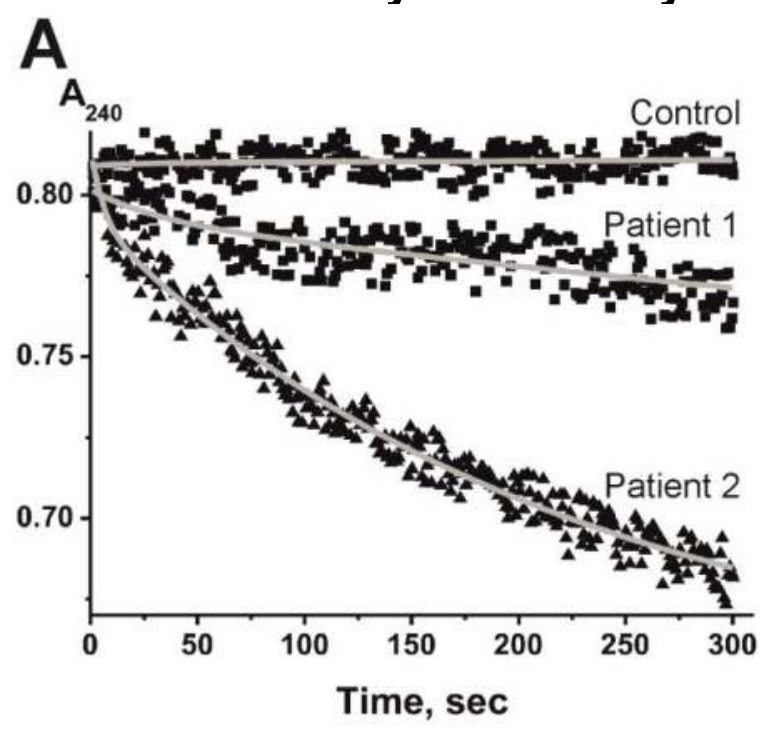

A - The decrease in $\mathrm{H} 2 \mathrm{O} 2$ absorbance at $240 \mathrm{~nm}$ (A240) in the presence of $\mathrm{lgG}$.
B

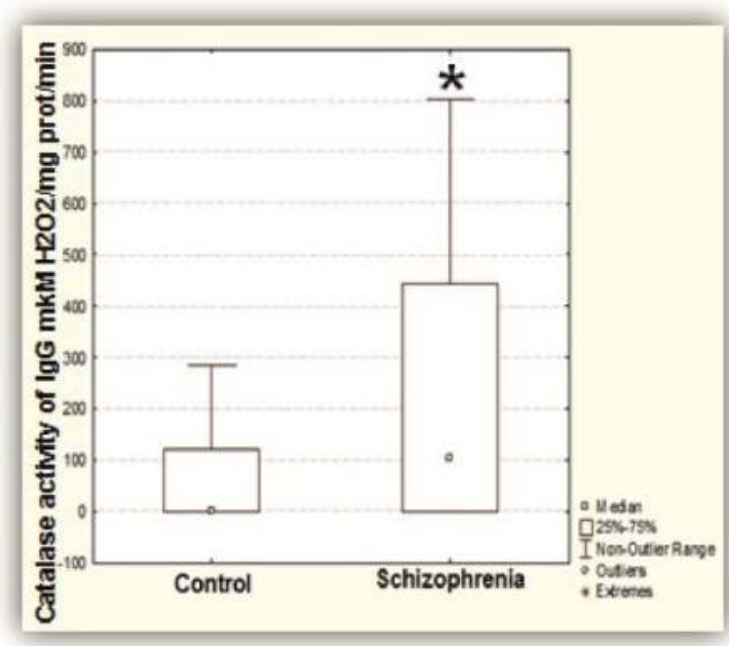

B - Level of catalase activity of IgG of healthy donors and schizophrenia patients.

\section{Catalase activity of abzymes is metal-dependent}
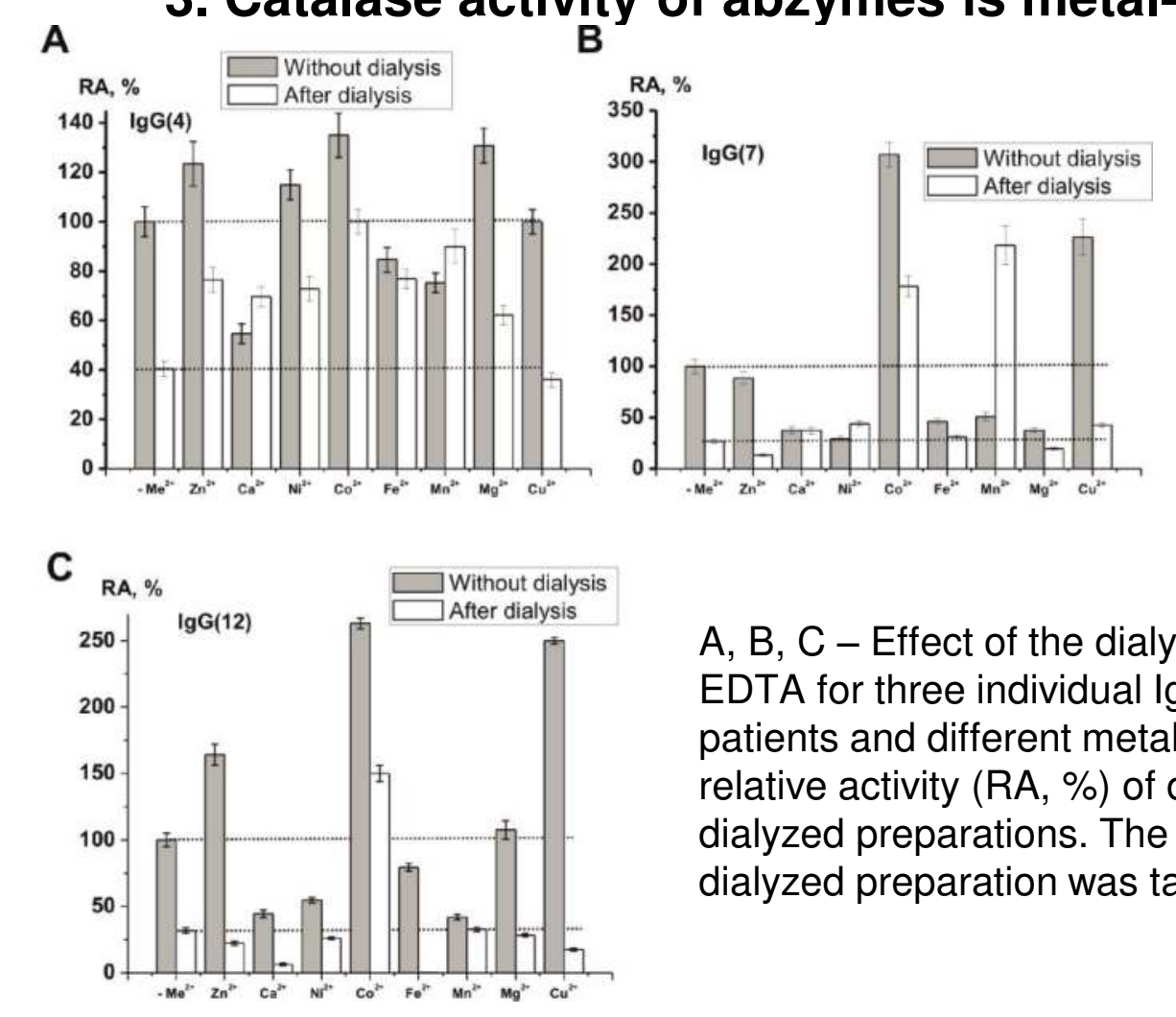

A, B, C - Effect of the dialysis of IgGs against EDTA for three individual IgG preparation of patients and different metal ions on the relative activity (RA, \%) of dialyzed and nondialyzed preparations. The RA of every nondialyzed preparation was taken for $100 \%$.

Table 1. Kinetic parameters of catalase activity of IgG abzymes and classical catalase-enzymes

\begin{tabular}{|c|c|c|c|c|}
\hline Parameters & IgG №1 & IgG №2 & $\begin{array}{c}\text { Erythrocyte } \\
\text { catalase }\end{array}$ & $\begin{array}{c}\text { Liver } \\
\text { catalase }\end{array}$ \\
\hline $\mathrm{K}_{\mathrm{m}}, \mathrm{mM}$ & $9.47 \pm 3.2$ & $7.8 \pm 4.5$ & $1.39 \times 10^{-3}$ & $1.29 \times 10^{-3}$ \\
\hline $\begin{array}{c}\mathrm{V}_{\text {max }}, \mathrm{mkM} \\
\mathrm{H}_{2} \mathrm{O}_{2} / \mathrm{min} / \mathrm{mg} \text { prot }\end{array}$ & $139.4 \pm 45.6$ & $119.1 \pm 49.3$ & - & - \\
\hline $\mathrm{k}_{\mathrm{cat}}, \mathrm{min}^{-1}$ & $15.85 \pm 5.3$ & $13.9 \pm 4.2$ & $2.22 \times 10^{7}$ & $2.27 \times 10^{7}$ \\
\hline
\end{tabular}

For more details see: Ermakov E. A. et al. Catalase activity of $\lg G$ antibodies from the sera of healthy donors and patients with schizophrenia // PloS One. - 2017. 12(9), e0183867 